\title{
Exercise Timing and Cancer Treatment: Avenues for Chronobiological Research
}

\author{
Lin Yang ${ }^{1,2}$, Philip Lewis ${ }^{3}$, and Yikyung Park ${ }^{4,5}$ \\ 'Department of Cancer Epidemiology and Prevention Research, CancerControl Alberta, Alberta Health Services, Calgary, Canada \\ 2Departments of Oncology and Community Health Sciences, University of Calgary, Calgary, Canada \\ ${ }^{3}$ Institute and Policlinic for Occupational Medicine, Environmental Medicine and Prevention Research, University Hospital of Cologne, Cologne, Germany \\ ${ }^{4}$ Division of Public Health Sciences, Department of Surgery, Washington University School of Medicine, St. Louis, MO, USA \\ ${ }^{5}$ Siteman Cancer Center, Washington University School of Medicine, St. Louis, MO, USA
}

Emerging evidence suggests that cancer chronotherapy, which involves timing drug administration in accordance with a patient's circadian/ internal time, can improve treatment tolerability and efficacy. However, as cancer, its treatments, and even hospitalization can cause circadian rhythm misalignment, cancer chronotherapy may be attenuated from the start. The adjunctive therapeutic strategy 'exercise' not only improves physical function and patient-reported outcomes, but specific effects are determined by the individual's internal time and it may act as a circadian time cue. Utilizing differentially timed exercise in terms of targeting time-specific homeostatic responses to the activity, accounting for the time of peak performance, and/or targeting potential circadian system effects of exercise could thus potentiate cancer chronotherapy or cancer treatment more generally. Herein, we briefly overview cancer chronotherapy and exercise medicine in oncology, highlight potential benefit of timed exercise in cancer treatment, and discuss research opportunities to assess these potentials benefits.

Key Words: Circadian; Chronobiology; Exercise oncology; Cancer treatment; Cancer therapy

Received: March 4, 2020 Accepted: April 8, 2020

Corresponding author: Lin Yang, PhD, Department of Cancer Epidemiology and Prevention Research, Cancer Research \& Analytics, CancerControl Alberta, Alberta Health Services, 5th Floor, Holy Cross Centre, Box ACB, 2210-2 St. SW, Calgary, AB T2S 3C3, Canada.

Tel: 1-403-698-8156, Fax: 1-403-264-2654, E-mail: lin.yang@ahs.ca

@ This is an Open Access article distributed under the terms of the Creative Commons Attribution Non-Commercial License (https://creativecommons.org/licenses/bync/4.0) which permits unrestricted non-commercial use, distribution, and reproduction in any medium, provided the original work is properly cited.

\section{INTRODUCTION}

The human circadian timing system temporally organizes myriad physiological parameters across 24 hours ('circa diem' from Latin meaning 'about a day') from the highs and lows observed in gene expression in cells to the highs and lows in physical and cognitive performance, and much more in between $[1,2]$. With particular relevance to cancer treatment wherein anticancer drugs can have aggressive, cell damaging, side effects, there are circadian highs and lows in cellular growth, DNA repair, and metabolism [2-5]. Indeed, differentially timed administration of anticancer drugs can elicit differential trade-offs between efficacy and side effects [6,7]. Consideration of circadian timing in anticancer drug administration is commonly referred to as cancer chronotherapy. Of course, cancer, its treatments, and hospitalization may also disrupt the circadian timing system and sleep of the patient $[6,8-10]$. If treatment efficacy and tolerability depends on the patient's circadian/internal time, disruptions to circadian timing or misalignment of circadian rhythms should be avoided. The goal must be to optimize circadian alignment in the patient to potentiate timed treatment and attenuate side effects. To this end, timed exercise may represent a valid and valuable, adjunctive, therapeutic strategy. Exercise is already encouraged as part of the treatment process and specified timing may, inter alia, exert chronobiological or diurnal effects that could aid cancer chronotherapy [11-15]. Herein, we briefly overview cancer chronotherapy and exercise medicine in oncology, highlight potential benefits of timed exercise in cancer treatment, and discuss research opportunities to assess these potential benefits.

\section{CANCER CHRONOTHERAPY}

Cancer chronotherapy involves specifically timed administration of anticancer drugs according to the individual's circadian/in- 
ternal time to improve treatment tolerance and efficacy $[6,16,17]$. That is to say, there will be an optimum time of day when trade-off between efficacy and side-effects are best based on whether particular circadian rhythms (for instance, cell growth, DNA repair, and metabolism [2-5]) are closer to their peaks or troughs in different tissues. In return for better-timed treatment, better treatment tolerability may lessen circadian disruptive effects of cancer, treatment, and hospitalization [6,8-10]. Furthermore, increased tolerability can facilitate further drug administration [7,16]. Based on experimental models, the chemotherapy tolerability can vary 2 - to 10-fold depending on the circadian timing of administration [16]. As of 2010, this pattern exists for at least 40 anticancer drugs; there are likely to be more in the meantime [16]. Evidently, timing can be important. Although the current application of cancer chronotherapy is limited, there have been promising findings from clinical trials. For instance, a meta-analysis of metastatic cancer patients receiving 5-fluorouacil, leucovorin, and oxaliplatin in chronomodulated infusions revealed significantly improved survival (median overall survival: 20.8 months) compared to those receiving conventional infusions (median overall survival: 17.5 months, p-value for difference $=0.009$ ) [18].

Clearly, cancer chronotherapies are focused on the internal time of the individual, and rightly so. But as cancer, its treatment, and hospitalization can have detrimental effects on a patient's sleep and circadian timing system, other factors that improve circadian rhythm alignment or lessen circadian disruption experienced by the patient may provide added benefit when combined with cancer chronotherapy.

\section{EXERCISE MEDICINE IN ONCOLOGY}

Exercise is a subset of physical activity that is planned, structured and repetitive with a purpose to improve health [19]. Recently, a consensus report called for increased exercise prescription in oncology and urged healthcare providers to actively promote and provide advice on physical activity, exercise, and appropriate exercise programs for cancer patients and survivors [11]. A body of literature supports the hypothesis that exercise can improve cancer treatment outcomes, quality of life and decrease risk of recurrence in cancer survivors [11]. To date, there have been approximately 700 exercise intervention studies conducted in the cancer survivor population $[11,20]$. Studies conducted in a mixed population, including cancer patients who were either receiving active treatment or have completed treatment, found physical activity/exercise improved survival among patients with malignant, recurrent glioma [21] and breast cancer [22].

In contrast, and despite growing evidence in support of the benefits of exercise in cancer survivors, few interventions have examined the effect of exercise on anticancer drug treatment tolerance and efficacy. There is promising data from animal studies indicating exercise may improve anticancer drug efficacies. Compared to mice treated with chemotherapy alone, mice treated with exercise plus chemotherapy presented with delayed tumor growth in mod- els of breast [23], melanoma [24], and pancreatic [24] cancers. A similar beneficial effect of exercise was observed to improve tamoxifen treatment in a mouse model of breast cancer [25]. In human studies, inconsistent results are reported: some indicate improvements [21,26-28], others indicate null findings [29-34].

Overall, several studies exploring adjunctive exercise interventions and treatment efficacy outcomes among cancer survivors indicate no adverse effect on treatment efficacy [28,29,33-35].

\section{EXERCISE TIMING AND CANCER TREATMENT}

The timing of exercise can affect peak performance levels, homeostatic responses to the activity, and potentially even the circadian timing of the individual [12-15,36-38]. As examples: 1) There is a diurnal rhythm to maximum achievable exercise performance levels with peaks typically observed in the afternoon and evening [36,37]; 2) Differential metabolic substrate utilization in response to exercise may occur depending on timing of exercise in relation to other exposures (such as feeding or fasting states) and/or concerning circadian/internal time [12,13,38]; 3) There is evidence in humans that exercise can phase shift circadian rhythm $[14,15]$. Conceivably, all three facets of timed exercise effects may be of benefit to the cancer patient. For instance, timed exercise may enhance circadian rhythm alignment in the cancer patient, which may lead to improved tolerability and efficacy of appropriately timed anticancer drugs (Figure 1). Wherein there is difficulty in performing exercise more generally, accounting for the time of day of the peak in performance may increase the exercise capability of the patient. Differential homeostatic responses to exercise depending on time of day and/or in relation to other exposures and homeostatic states (e.g., metabolic substrate utilization) may be useful in constructing strategies against e.g., cancer-induced cachexia [12,39].

\section{OPPORTUNITIES FOR RESEARCH}

Given the large and increasing burden of cancer, investigation of the different facets of timed exercise as adjunctive therapeutic strategies are warranted. To this end, we provide recommendations: Firstly, exercise interventions in cancer co-treatment should include using parameters of treatment efficacy as primary endpoints such as tumor response [Response Evaluation Criteria in Solid Tumors (RECIST)] in solid tumors, CT tumor density for tumors treated with molecular targeting agent, and immune-checkpoint blockade and atypical response patterns for cancer immunotherapy, etc [40]. Secondly, both observational and interventional studies should capture the circadian timing (or at least time of day) of exercise in addition to individual chronotype information (indicative of whether peaks/troughs in rhythms are likely to occur earlier or later in the day). Ideally, this will be done using wearable accelerometers. Indeed, wearables such as Actigraph (Pensacola, FL, USA) [41], GENEActiv (Cambridge, UK) [42], and Axivity (Newcastle, UK) 


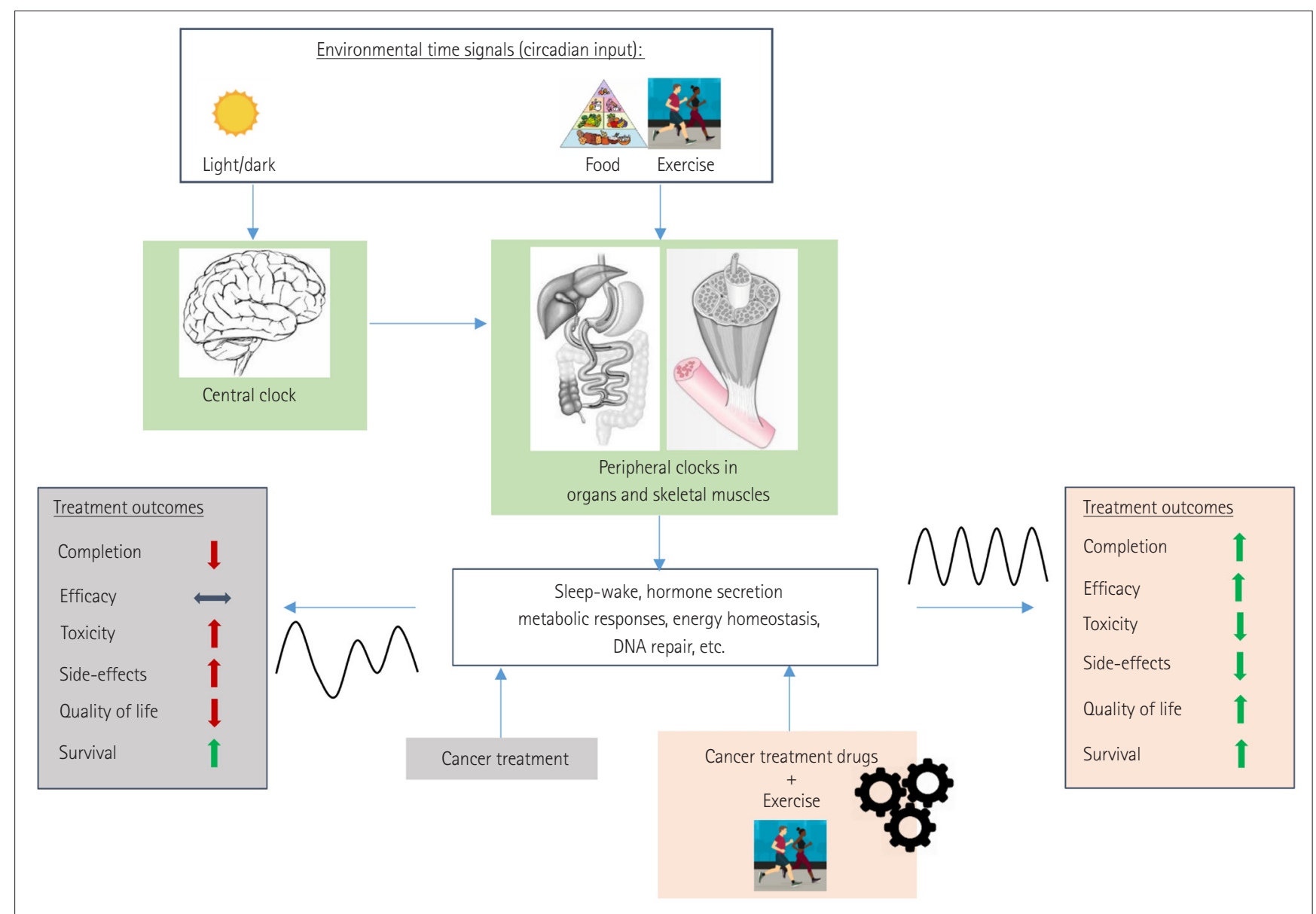

Figure 1. Hypothesized pathway through which timed exercise may influence cancer chronotherapy toxicities and efficacies.

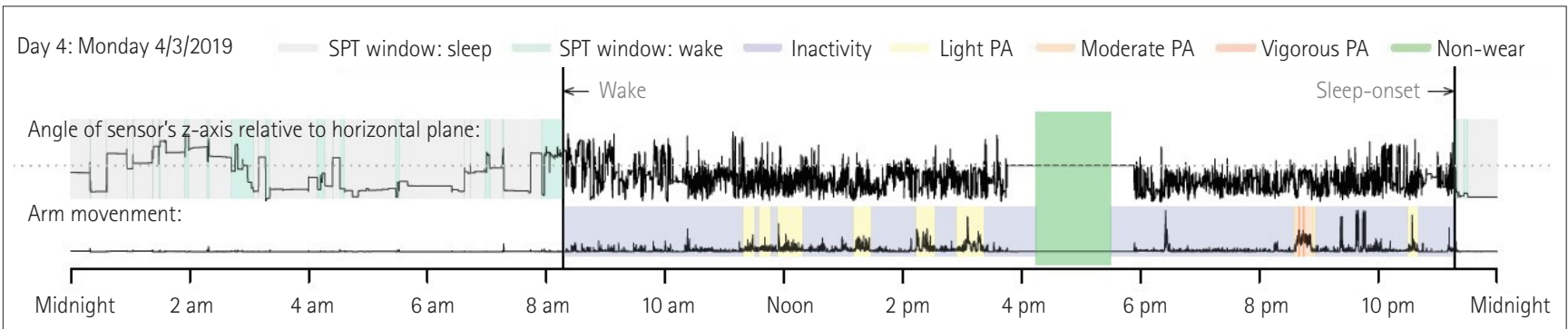

Figure 2. An example of the visual summary of accelerometer measured 24-hour temporal rest-activity patterns. SPT: sleep period time, PA: physical activity. Data processed by GGIR R-package [46], available online: https://cran.r-project.org/web/packages/GGIR/vignettes/GGIR.html.

[43] can be used to capture data related to intensity, frequency, duration, and timing of physical activity (Figure 2). It is expected that detailed drug treatment data including dose, frequency, time of administration, completion rate, dose reductions, side effects, specific treatment responses, and patient-reported outcomes should also be collected. Whether observational or interventional in design, such studies would add to the knowledge base on whether specifically timed exercise could be a viable and valuable adjunctive therapeutic strategy against cancer. Thirdly, it would be beneficial for studies investigating the circadian-associated effects of exercise on cancer treatment to collect biological samples. Biological samples will provide opportunities to study pathways associated with physiological responses of the human body to specifically timed exercise during cancer treatments.

Additionally, existing studies that have collected accelerometer data among cancer survivors or patients undergoing cancer treatment could potentially be revisited to generate parameters that facilitate exploratory analyses. Finally, beyond exercise but in a similar vein, studies involving the timing of food may be warranted given that food timing may also affect the human circadian system and circadian rhythm may also affect the homeostatic response to food (i.e., the fate nutrients) $[44,45]$. 


\section{CONCLUSION}

Research activities in exercise oncology recently began to investigate the effect of exercise on cancer treatment efficacy. Given that timing of exercise can have a diverse array of potential effects with relevance to cancer patients, studies that incorporate timing are warranted and may lead to innovative strategies in the fight against cancer.

\section{Acknowledgments}

None

\section{Conflicts of Interest}

The authors have no potential conflicts of interest to disclose.

\section{Author Contributions}

Conceptualization: Lin Yang, Yikyung Park. Data curation: Lin Yang. Investigation: Lin Yang, Philip Lewis, Yikyung Park. Methodology: Lin Yang, Philip Lewis, Yikyung Park. Project administration: Lin Yang. Resources: Lin Yang. Supervision: Yikyung Park. Visualization: Lin Yang, Yikyung Park. Writing_original draft: Lin Yang. Writing_-review \& editing: Lin Yang, Philip Lewis, Yikyung Park.

\section{ORCID iD}

Lin Yang (1)

https://orcid.org/0000-0002-1698-6666

\section{REFERENCES}

1. Takahashi JS. Molecular architecture of the circadian clock in mammals. In: Sassone-Corsi P, Christen Y, editors. A time for metabolism and hormones. Cham (CH): Springer; 2016, p. 13-24.

2. Pittendrigh CS. Circadian rhythms and the circadian organization of living systems. Cold Spring Harb Symp Quant Biol 1960;25:159-184.

3. Yang Y, Adebali O, Wu G, Selby CP, Chiou YY, Rashid N, et al. CisplatinDNA adduct repair of transcribed genes is controlled by two circadian programs in mouse tissues. Proc Natl Acad Sci U S A 2018;115:E4777-E4785.

4. Dakup PP, Porter KI, Little AA, Gajula RP, Zhang H, Skornyakov E, et al. The circadian clock regulates cisplatin-induced toxicity and tumor regression in melanoma mouse and human models. Oncotarget 2018;9:1452414538.

5. Sulli G, Manoogian ENC, Taub PR, Panda S. Training the circadian clock, clocking the drugs, and drugging the clock to prevent, manage, and treat chronic diseases. Trends Pharmacol Sci 2018;39:812-827.

6. Lévi F. Circadian chronotherapy for human cancers. Lancet Oncol 2001;2: 307-315.

7. Lévi F. Cancer chronotherapy. J Pharm Pharmacol 1999;51:891-898.

8. Ancoli-Israel S, Liu L, Marler MR, Parker BA, Jones V, Sadler GR, et al. Fatigue, sleep, and circadian rhythms prior to chemotherapy for breast cancer. Support Care Cancer 2006;14:201-209.

9. Chen HM, Wu YC, Tsai CM, Tzeng JI, Lin CC. Relationships of circadian rhythms and physical activity with objective sleep parameters in lung cancer patients. Cancer Nurs 2015;38:215-223.

10. Milani RV, Bober RM, Lavie CJ, Wilt JK, Milani AR, White CJ. Reducing hospital toxicity: impact on patient outcomes. Am J Med 2018;131:961966.

11. Schmitz KH, Campbell AM, Stuiver MM, Pinto BM, Schwartz AL, Morris GS, et al. Exercise is medicine in oncology: engaging clinicians to help patients move through cancer. CA Cancer J Clin 2019;69:468-484.

12. Gabriel BM, Zierath JR. Circadian rhythms and exercise: re-setting the clock in metabolic disease. Nat Rev Endocrinol 2019;15:197-206.

13. Sato S, Basse AL, Schönke M, Chen S, Samad M, Altıntaş A, et al. Time of exercise specifies the impact on muscle metabolic pathways and systemic energy homeostasis. Cell Metab 2019;30:92-110.

14. Lewis P, Korf HW, Kuffer L, Groß JV, Erren TC. Exercise time cues (zeitgebers) for human circadian systems can foster health and improve performance: a systematic review. BMJ Open Sport Exerc Med 2018;4:e000443.

15. Youngstedt SD, Elliott JA, Kripke DF. Human circadian phase-response curves for exercise. J Physiol 2019;597:2253-2268.

16. Lévi F, Okyar A, Dulong S, Innominato PF, Clairambault J. Circadian timing in cancer treatments. Annu Rev Pharmacol Toxicol 2010;50:377-421.

17. Smolensky MH, Peppas NA. Chronobiology, drug delivery, and chronotherapeutics. Adv Drug Deliv Rev 2007;59:828-851.

18. Giacchetti S, Dugué PA, Innominato PF, Bjarnason GA, Focan C, Garufi C, et al. Sex moderates circadian chemotherapy effects on survival of patients with metastatic colorectal cancer: a meta-analysis. Ann Oncol 2012;23:31103116.

19. Caspersen CJ, Powell KE, Christenson GM. Physical activity, exercise, and physical fitness: definitions and distinctions for health-related research. Public Health Rep 1985;100:126-131.

20. Christensen JF, Simonsen C, Hojman P. Exercise training in cancer control and treatment. Compr Physiol 2018;9:165-205.

21. Ruden E, Reardon DA, Coan AD, Herndon JE 2nd, Hornsby WE, West M, et al. Exercise behavior, functional capacity, and survival in adults with malignant recurrent glioma. J Clin Oncol 2011;29:2918-2923.

22. Chen X, Lu W, Zheng W, Gu K, Matthews CE, Chen Z, et al. Exercise after diagnosis of breast cancer in association with survival. Cancer Prev Res (Phila) 2011;4:1409-1418.

23. Betof AS, Lascola CD, Weitzel D, Landon C, Scarbrough PM, Devi GR, et al. Modulation of murine breast tumor vascularity, hypoxia and chemotherapeutic response by exercise. J Natl Cancer Inst 2015;107:djv040.

24. Schadler KL, Thomas NJ, Galie PA, Bhang DH, Roby KC, Addai P, et al. Tumor vessel normalization after aerobic exercise enhances chemotherapeutic efficacy. Oncotarget 2016;7:65429-65440.

25. Khori V, Amani Shalamzari S, Isanejad A, Alizadeh AM, Alizadeh S, Khodayari S, et al. Effects of exercise training together with tamoxifen in reducing mammary tumor burden in mice: possible underlying pathway of miR21. Eur J Pharmacol 2015;765:179-187.

26. Courneya KS, Friedenreich CM, Franco-Villalobos C, Crawford JJ, Chua N, Basi S, et al. Effects of supervised exercise on progression-free survival in lymphoma patients: an exploratory follow-up of the HELP Trial. Cancer Causes Control 2015;26:269-276.

27. Wiskemann J, Kuehl R, Dreger P, Huber G, Kleindienst N, Ulrich CM, et al. Physical Exercise Training versus Relaxation in Allogeneic stem cell transplantation (PETRA Study)-Rationale and design of a randomized trial to evaluate a yearlong exercise intervention on overall survival and side-effects after allogeneic stem cell transplantation. BMC Cancer 2015;15:619.

28. Hayes SC, Steele ML, Spence RR, Gordon L, Battistutta D, Bashford J, et al. Exercise following breast cancer: exploratory survival analyses of two randomised, controlled trials. Breast Cancer Res Treat 2018;167:505-514.

29. Courneya KS, Sellar CM, Stevinson C, McNeely ML, Peddle CJ, Friedenreich CM, et al. Randomized controlled trial of the effects of aerobic exercise on physical functioning and quality of life in lymphoma patients. J Clin Oncol 2009;27:4605-4612.

30. Oldervoll LM, Loge JH, Lydersen S, Paltiel H, Asp MB, Nygaard UV, et al. Physical exercise for cancer patients with advanced disease: a randomized controlled trial. Oncologist 2011;16:1649-1657.

31. Christensen JF, Simonsen C, Banck-Petersen A, Thorsen-Streit S, Herrstedt A, Djurhuus SS, et al. Safety and feasibility of preoperative exercise training during neoadjuvant treatment before surgery for adenocarcinoma of the gastro-oesophageal junction. BJS Open 2018;3:74-84.

32. Rief H, Bruckner T, Schlampp I, Bostel T, Welzel T, Debus J, et al. Resistance training concomitant to radiotherapy of spinal bone metastases - survival and prognostic factors of a randomized trial. Radiat Oncol 2016;11:97.

33. Chiarotto JA, Akbarali R, Bellotti L, Dranitsaris G. A structured group exercise program for patients with metastatic cancer receiving chemotherapy and CTNNB1 ( $\beta$-catenin) as a biomarker of exercise efficacy. Cancer Manag Res 2017;9:495-501.

34. Dhillon HM, Bell ML, van der Ploeg HP, Turner JD, Kabourakis M, Spen- 
cer L, et al. Impact of physical activity on fatigue and quality of life in people with advanced lung cancer: a randomized controlled trial. Ann Oncol 2017;28:1889-1897.

35. Rief H, Omlor G, Akbar M, Welzel T, Bruckner T, Rieken S, et al. Feasibility of isometric spinal muscle training in patients with bone metastases under radiation therapy - first results of a randomized pilot trial. BMC Cancer 2014;14:67.

36. Chtourou H, Souissi N. The effect of training at a specific time of day: a review. J Strength Cond Res 2012;26:1984-2005.

37. Facer-Childs E, Brandstaetter R. The impact of circadian phenotype and time since awakening on diurnal performance in athletes. Curr Biol 2015;25: 518-522.

38. Vieira AF, Costa RR, Macedo RC, Coconcelli L, Kruel LF. Effects of aerobic exercise performed in fasted $\mathrm{v}$. fed state on fat and carbohydrate metabolism in adults: a systematic review and meta-analysis. Br J Nutr 2016;116:11531164.

39. Tsoli M, Schweiger M, Vanniasinghe AS, Painter A, Zechner R, Clarke S, et al. Depletion of white adipose tissue in cancer cachexia syndrome is associated with inflammatory signaling and disrupted circadian regulation. PLoS One 2014;9:e92966.

40. Nishino M. Tumor response assessment for precision cancer therapy: response evaluation criteria in solid tumors and beyond. Am Soc Clin Oncol
Educ Book 2018;38:1019-1029.

41. Santos-Lozano A, Santín-Medeiros F, Cardon G, Torres-Luque G, Bailón R, Bergmeir C, Ruiz JR, et al. Actigraph GT3X: validation and determination of physical activity intensity cut points. Int J Sports Med 2013;34:975-982.

42. Duncan MJ, Rowlands A, Lawson C, Leddington Wright S, Hill M, Morris $\mathrm{M}$, et al. Using accelerometry to classify physical activity intensity in older adults: what is the optimal wear-site? Eur J Sport Sci 2019 Nov 26 [Epub]. Available at: https://doi.org/10.1080/17461391.2019.1694078]

43. Crowley P, Skotte J, Stamatakis E, Hamer M, Aadahl M, Stevens ML, et al. Comparison of physical behavior estimates from three different thighworn accelerometers brands: a proof-of-concept for the Prospective Physical Activity, Sitting, and Sleep consortium (ProPASS). Int J Behav Nutr Phys Act 2019;16:65.

44. Lewis P, Oster H, Korf HW, Foster RG, Erren TC. Food as a circadian time cue - evidence from human studies. Nat Rev Endocrinol 2020;16:213-223.

45. Srour B, Plancoulaine S, Andreeva VA, Fassier P, Julia C, Galan P, et al. Circadian nutritional behaviours and cancer risk: new insights from the NutriNetsanté prospective cohort study. Int J Cancer 2018;143:2369-2379.

46. Van Hees V, Fang Z, Zhao J, Heywood J, Mirkes E, Sabia S, et al. GGIR: raw accelerometer data analysis. R package version 1.11-0, 2019. Available at: https://CRAN.R-project.org/package=GGIR. 